16,17

\title{
Равновесные структуры из углеродных алмазоподобных кластеров и их упругие свойства
}

\author{
(ㄱ Д.С. Лисовенко ${ }^{1}$ Ю.А. Баимова ${ }^{2,3}$, Л.Х. Рысаева ${ }^{2}$, В.А. Городцов ${ }^{1}$, С.В. Дмитриев ${ }^{2,4}$ \\ ${ }^{1}$ Институт проблем механики им. А.Ю. Ишлинского РАН, \\ Москва, Россия \\ ${ }^{2}$ Институт проблем сверхпластичности металлов РАН, \\ Уфра, Россия \\ ${ }^{3}$ Институт физики металлов им. М.Н. Михеева УрО РАН, \\ Екатеринбург, Россия \\ ${ }^{4}$ Национальный исследовательский Томский государственный университет, \\ Томск, Россия \\ E-mail: lisovenk@ipmnet.ru \\ (Поступила в Редакцию 21 марта 2016 г. \\ В окончательной редакции 7 сентября 2016 г.)
}

Методом молекулярно-динамического моделирования изучены трехмерные углеродные алмазоподобные фазы, состоящие из $s p^{3}$-гибридизированных атомов, полученные сшивкой каркасов фуллереноподобных молекул. Для восьми кубических и одной гексагональной алмазоподобных фаз на основе фуллереноподобных молекул четырех типов найдены равновесные конфигурации и рассчитаны упругие константы. Результаты, полученные методом молекулярной динамики, использованы для аналитических расчетов упругих характеристик исследованных алмазоподобных фаз с кубической и гексагональной анизотропией. Найдено, что три из рассмотренных фаз при определенном выборе оси растяжения имеют отрицательный коэффициент Пуассона, т.е. являются частичными ауксетиками. Проанализирована изменчивость технических коэффициентов упругости (модуля Юнга, коэффициента Пуассона, модуля сдвига и объемного модуля).

С.В. Дмитриев благодарит за финансовую поддержку РНФ (грант № 14-13-00982). Ю.А. Баимова выражает благодарность за стипендию Президента РФ молодым ученым и аспирантам (СП-4037.2015.1). Л.Х. Рысаева благодарит за финансовую поддержку РФФИ (грант № 16-32-00483-мол_а).

Анализ упругих свойств структур с кубической анизотропией из углеродных алмазоподобных кластеров проведен Д.С. Лисовенко и В.А. Городцовым в рамках гранта РФФИ № 16-01-00325.

DOI: 10.21883/FTT.2017.04.44286.100

\section{1. Введение}

Углеродные алмазоподобные фазы (УАФ) - это фазы, состоящие из углеродных атомов, в которых каждый атом, как в алмазной структуре, образует четыре $\sigma$-связи с соседними атомами [1]. При этом решетка таких алмазоподобных фаз отличается от решетки алмаза. Основой для создания алмазоподобных фаз могут служить различные полиморфы углерода: графен, фуллереноподобные молекулы, трубчатые структуры на основе углерода. Алмазоподобные фазы, составной единицей которых являются фуллереноподобные молекулы, получили название „фуллераны“, материал, состоящий из листов графена, - „графан“, а из нанотрубок „тубулан“ [1]. Наряду с наноалмазами [2] такие структуры вызывают большой интерес исследователей. К настоящему времени экспериментально синтезировано и теоретически исследовано несколько углеродных алмазоподобных фаз, например полимеризованный кубический фуллерит $\mathrm{C}_{24}[3,4]$, углеродная фаза $\mathrm{C}_{8}$ высокой плотности [5] и т.д. В ряде теоретических работ довольно полно представлены характеристики различных алмазоподобных фаз и их классификация [6-9]. В работе [10] впервые было предложено получать наноал- мазную фазу ковалентно связанных полимеризованных нанотрубок $(4,0)$. В результате проведенных расчетов установлено, что кристалл из полимеризованных нанотрубок должен быть полупроводником с шириной запрещенной зоны $3.18 \mathrm{eV}$ [10]. Методами молекулярной динамики (МД) были исследованы структуры карбиноидных слоев, карбиноидных нанотрубок и карбинофуллеренов и получены их основные характеристики [11]. Структурные характеристики кристаллов, получаемых из полиморфов графена, были исследованы в [12]. УАФ и их свойства изучались ранее в работах [13-15]. Методом МД рассчитаны равновесные состояния кластеров фуллереноподобных молекул $\mathrm{C}_{24}$ и $\mathrm{C}_{48}[16,17]$.

Наиболее важной областью применения УАФ является покрытие различных поверхностей в биомедицине [18], при защите материалов и устройств от внешних воздействий [19], легко изнашиваемых материалов [20], а также в антифрикционных покрытиях [21]. Получение таких материалов можно осуществить гораздо легче и дешевле по сравнению с алмазами.

Анализ упругих свойств однослойных углеродных нанотрубок показал, что они могут иметь отрицательный коэффициент Пуассона [22-24]. У многих анизотропных 
наноматериалов различных кристаллических систем (кубической, гексагональной, тетрагональной и т.п.) также обнаруживается отрицательный коэффициент Пуассона при растяжениях в определенных направлениях [25-38]. На данный момент известен список из более чем четырехсот материалов с отрицательным коэффициентом Пуассона (ауксетиков). При этом более трехсот таких ауксетиков имеют кубическую анизотропию [25,37]. Ранее был проведен анализ упругих постоянных некоторых алмазоподобных фаз и фуллерита [39-42]. Упругие модули монокристаллического $\mathrm{C}_{60}$ были определены на основе измерения скоростей ультразвука и составили величины порядка $c_{11} \sim 15 \mathrm{GPa}, c_{12} \sim 9 \mathrm{GPa}$ и $c_{44} \sim 6 \mathrm{GPa}$ [39], исследованы релаксационный вклад [40] и температурное поведение [41,42] модулей упругости. Значения объемных модулей алмазоподобных фаз на основе фуллереноподобных молекул были рассчитаны в работе [13]. Показано, что величина модулей изменяется от 141.2 до $350.5 \mathrm{GPa}$, что уступает соответствующему значению объемного модуля кубического алмаза. Структурные и энергетические характеристики различных алмазоподобных фаз активно исследуются в последние годы, однако многие их свойства остаются малоизученными и требуют дальнейших исследований.

В настоящей работе рассматриваются алмазоподобные фазы с кубической, гексагональной и тетрагональной анизотропией, равновесное состояние которых исследовано методом МД. Данные, полученные с помощью атомистического моделирования, используются для анализа упругих характеристик.

\section{2. Методика моделирования}

Устойчивые УАФ обсуждались в [1]; исходя из представленных там расчетов в настоящей работе исследуются алмазоподобные фазы, состоящие из фуллереноподобных молекул. Согласно [1,13], один и тот же фуллерен можно использовать для создания различных алмазоподобных фаз, поскольку могут различаться методы сшивки молекул. Название структур соответствует типу соединения двух фуллеренов между собой. Например, структура с обозначением А образуется сшивкой ковалентными связями, а с обозначением В - совмещением атомов углерода (рис. 1). Исходные параметры алмазоподобных фаз СА1, СА2, СА3, СА4, СА5, СА6, СА7, СА8, СА9 и СВ приведены в работах $[1,13]$. Фуллереноподобные молекулы, из которых были составлены исследуемые структуры, показаны на рис. 1: $\mathrm{C}_{4}$, $\mathrm{C}_{6}, \mathrm{C}_{8}, \mathrm{C}_{16}, \mathrm{C}_{24}$ и $\mathrm{C}_{48}$. Из десяти УАФ, исследуемых в работе, составным элементом которых является фуллереноподобная молекула, восемь (СА1, СА3, СА4, СА6, CA7, СА8, СА9, СВ) имеют кубическую анизотропию, фаза СА2 имеет гексагональную анизотропию, а фаза СА5 - тетрагональную [1].

Для получения равновесных структур алмазоподобных фаз и оценки их характеристик был использован программный пакет МД-моделирования LAMMPS [43],
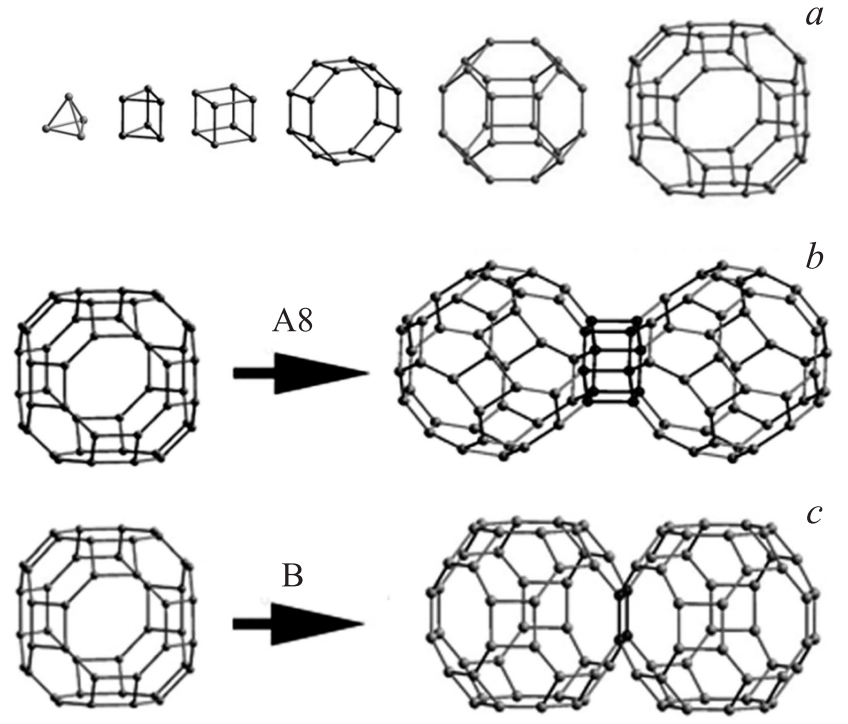

Рис. 1. Фуллереноподобные молекулы $\mathrm{C}_{4}, \mathrm{C}_{6}, \mathrm{C}_{8}, \mathrm{C}_{16}, \mathrm{C}_{24}$, $\mathrm{C}_{48}$ (слева направо) (a) и два способа соединения фуллереноподобных молекул в кластер $(b, c)$.

где для описания межатомного взаимодействия используется потенциал AIREBO [44]. В данном потенциале энергия связи задается как

$$
E_{b}=\sum_{j} \sum_{j(j>1)}\left[V_{R}\left(r_{i j}\right)-\bar{B}_{i j} V_{A}\left(r_{i j}\right)\right],
$$

где $r_{i j}$ - расстояние между атомами $i$ и $j, \bar{B}_{i j}-$ эмпирическая функция, которая была определена в работе [45], $V_{R}$ и $V_{A}$ - функции, отвечающие за притяжение и отталкивание. $V_{R}$ и $V_{A}$ задаются выражениями

$$
\begin{aligned}
& V_{R}\left(r_{i j}\right)=\frac{D^{(e)}}{S-1} e^{-\sqrt{2 S \beta}\left(r_{i j}-R^{(e)}\right)} f_{c}\left(r_{i j}\right), \\
& V_{A}\left(r_{i j}\right)=\frac{D^{(e)} S}{S-1} e^{-\sqrt{2 S \beta}\left(r_{i j}-R^{(e)}\right)} f_{c}\left(r_{i j}\right),
\end{aligned}
$$

где величины $D^{(e)}, S, \beta, R^{(e)}$ и функция $f_{c}$ были определены в [45].

Такой потенциал хорошо зарекомендовал себя для моделирования углеродных и углеводородных структур, хорошо воспроизводит свойства ковалентной связи между атомами углерода и широко используется для моделирования одно- и многослойных листов графена $[46,47]$, смятого графена $[48,49]$, фуллеренов [50] и т. п. Данные, полученные с помощью этого потенциала, хорошо согласуются с экспериментальными результатами и расчетами $a b$ initio.

Для получения равновесного состояния рассматриваемых алмазоподобных фаз выполнялась минимизация энергий с последующей релаксацией. Релаксация системы проводилась с помощью термостата Носе-Хувера. Ньютоновские уравнения движения атомов интегрировались по алгоритму Верле четвертого порядка. Далее 
проводился расчет модулей податливости $s_{i j}$ и модулей жесткости $c_{i j}$. С этой целью к ячейке моделирования прикладывалась малая однородная деформация с одной ненулевой компонентой и вычислялись возникающие при этом напряжения, которые затем подставлялись в закон Гука для расчета искомых констант.

\section{3. Результаты моделирования}

3.1. Равновесные структ уры. Примеры равновесных фаз из кластеров углеродных алмазоподобных молекул могут быть найдены в $[1,13]$ и поэтому в настоящей работе не приведены. Далее алмазоподобные фазы описаны для десяти исследованных структур методом релаксации до состояния с минимальной энергией.

Для расчета модулей податливости $s_{i j}$ к ячейке моделирования прикладывалось линейно увеличивающееся во времени напряжение с одной ненулевой компонентой и вычислялись возникающие при этом деформации. Оказалось, что для деформаций менее $2 \%$ кривые напряжение-деформация практически линейны; по наклонам данных кривых из закона Гука рассчитывались искомые константы $s_{i j}$.

Для кубического кристалла достаточно рассчитать только три модуля податливости из выражения

$$
\left(\begin{array}{l}
\varepsilon_{1} \\
\varepsilon_{2} \\
\varepsilon_{3} \\
\varepsilon_{4} \\
\varepsilon_{5} \\
\varepsilon_{6}
\end{array}\right)=\left(\begin{array}{cccccc}
s_{11} & s_{12} & s_{12} & 0 & 0 & 0 \\
s_{12} & s_{11} & s_{12} & 0 & 0 & 0 \\
s_{12} & s_{12} & s_{11} & 0 & 0 & 0 \\
0 & 0 & 0 & s_{44} & 0 & 0 \\
0 & 0 & 0 & 0 & s_{44} & 0 \\
0 & 0 & 0 & 0 & 0 & s_{44}
\end{array}\right)\left(\begin{array}{l}
\sigma_{1} \\
\sigma_{2} \\
\sigma_{3} \\
\sigma_{4} \\
\sigma_{5} \\
\sigma_{6}
\end{array}\right)
$$

Здесь подразумевается соответствие $\varepsilon_{x x} \rightarrow \varepsilon_{1}, \varepsilon_{y y} \rightarrow \varepsilon_{2}$, $\varepsilon_{z z} \rightarrow \varepsilon_{3}, \quad 2 \varepsilon_{y z} \rightarrow \varepsilon_{4}, \quad 2 \varepsilon_{x z} \rightarrow \varepsilon_{5}, \quad 2 \varepsilon_{x y} \rightarrow \varepsilon_{6}, \quad \sigma_{x x} \rightarrow \sigma_{1}$, $\sigma_{y y} \rightarrow \sigma_{2}, \sigma_{z z} \rightarrow \sigma_{3}, \sigma_{y z} \rightarrow \sigma_{4}, \sigma_{x z} \rightarrow \sigma_{5}, \sigma_{x y} \rightarrow \sigma_{6} ; s_{11}, s_{12}$ и $s_{44}$ - матричные модули податливости кубического кристалла. Для гексагональной анизотропии закон Гука выглядит следующим образом:

$$
\left(\begin{array}{c}
\varepsilon_{1} \\
\varepsilon_{2} \\
\varepsilon_{3} \\
\varepsilon_{4} \\
\varepsilon_{5} \\
\varepsilon_{6}
\end{array}\right)=\left(\begin{array}{cccccc}
s_{11} & s_{12} & s_{13} & 0 & 0 & 0 \\
s_{12} & s_{11} & s_{13} & 0 & 0 & 0 \\
s_{13} & s_{13} & s_{33} & 0 & 0 & 0 \\
0 & 0 & 0 & s_{44} & 0 & 0 \\
0 & 0 & 0 & 0 & s_{44} & 0 \\
0 & 0 & 0 & 0 & 0 & s_{66}
\end{array}\right)\left(\begin{array}{l}
\sigma_{1} \\
\sigma_{2} \\
\sigma_{3} \\
\sigma_{4} \\
\sigma_{5} \\
\sigma_{6}
\end{array}\right)
$$

где $s_{11}, s_{33}, s_{44}, s_{12}, s_{13}, s_{66}=2\left(s_{11}-s_{12}\right)$ - матричные модули податливости гексагонального кристалла.

Модули жесткости для фаз с кубической анизотропией были рассчитаны с учетом модулей податливости следующим образом:

$$
\begin{aligned}
& c_{11}+c_{12}=\frac{s_{11}}{\left(s_{11}-s_{12}\right)\left(s_{11}+s_{12}\right)}, \\
& c_{11}-c_{12}=\frac{1}{s_{11}-s_{12}}, \quad c_{44}=\frac{1}{s_{44}},
\end{aligned}
$$

Таблица 1. Значения модулей податливости $s_{i j}$ и модулей жесткости $c_{i j}$ для углеродных алмазоподобных структур с кубической анизотропией

\begin{tabular}{l|c|c|c|c|c|c}
\hline УАФ & $\begin{array}{c}s_{11}, \\
\mathrm{TPa}^{-1}\end{array}$ & $\begin{array}{c}s_{44}, \\
\mathrm{TPa}^{-1}\end{array}$ & $\begin{array}{c}s_{12}, \\
\mathrm{TPa}^{-1}\end{array}$ & $\begin{array}{c}c_{11}, \\
\mathrm{GPa}\end{array}$ & $\begin{array}{c}c_{44}, \\
\mathrm{GPa}\end{array}$ & $\begin{array}{c}c_{12}, \\
\mathrm{GPa}\end{array}$ \\
\hline $\mathrm{CA3}$ & 1.87 & 2.496 & -0.44 & 625 & 401 & 192 \\
$\mathrm{CA} 4$ & 1.87 & 9.64 & -0.437 & 624 & 104 & 190 \\
$\mathrm{CA6}$ & 0.948 & 8.66 & -0.072 & 1068 & 115 & 87.8 \\
$\mathrm{CA} 7$ & 8.12 & 3.64 & 3.82 & 455 & 262 & 370 \\
$\mathrm{CA} 8$ & 1.67 & 5.91 & -0.299 & 650 & 169 & 142 \\
CA9 & 3.73 & 7.31 & -0.90 & 316 & 137 & 101 \\
CB & 5.53 & 10 & -2.0 & 306 & 99.9 & 174
\end{tabular}

а для фаз с гексагональной анизотропией - как

$$
\begin{gathered}
c_{11}+c_{12}=\frac{s_{33}}{s}, \quad c_{11}-c_{12}=\frac{1}{s_{11}-s_{12}}, \\
c_{13}=-\frac{s_{13}}{s}, \quad c_{33}=\frac{\left(s_{11}+s_{12}\right)}{s}, \\
c_{44}=\frac{1}{s_{44}}, \quad c_{66}=\frac{1}{s_{66}},
\end{gathered}
$$

где $s=s_{33}\left(s_{11}+s_{12}\right)-2 s_{13}^{2}>0$.

Аналогично для уточнения полученных модулей упругости и модулей податливости был применен другой метод расчета: к ячейке моделирования прикладывалась линейно увеличивающаяся со временем деформация с одной ненулевой компонентой и вычислялись возникающие при этом напряжения. Отсюда рассчитывались модули жесткости, а с их использованием - модули податливости. Сравнение результатов, полученных разными методами, показало, что первый метод расчета дает более адекватные результаты. Различие результатов, полученных двумя, казалось бы, подобными методами, можно объяснить тем, что рассматриваемые УАФ поразному реагируют на различные виды нагружения. Параметры более устойчивых из УАФ могут быть рассчитаны любым из представленных способов.

Полученные значения для алмазоподобных фаз с кубической анизотропией (СА3, СА4, СА6, СА7, СА8, СА9 и СВ) приведены в табл. 1. Для гексагональной фазы СА2, основываясь на аналогичном моделировании, имеем следующие значения модулей податливости: $s_{11}=2.515 \mathrm{TPa}^{-1}, s_{33}=1.92 \mathrm{TPa}^{-1}$, $s_{44}=18.4 \mathrm{TPa}^{-1}, s_{12}=0.0906 \mathrm{TPa}^{-1}, s_{13}=-0.3625 \mathrm{TPa}^{-1}$ и $s_{66}=5.94 \mathrm{TPa}^{-1}$. Используя связь между модулями податливости и модулями жесткости, получаем $c_{11}=409 \mathrm{GPa}, c_{33}=550 \mathrm{GPa}, c_{44}=54.4 \mathrm{GPa}$, $c_{12}=-3.705 \mathrm{GPa}, c_{13}=76.5 \mathrm{GPa} \mathrm{и} c_{66}=168 \mathrm{GPa}$.

Деформация структуры СА5 выявила ее неустойчивость. Даже малая деформация (до 0.01\%) приводит к перестройке структуры с разрывом некоторых связей. Аналогично фаза СА1 показала себя как малоустойчивая в динамических условиях. Модули упругости, полученные для этой фазы, не соответствуют действительности, 
и получение достоверных результатов для этой структуры требует дальнейших исследований.

3.2. Упругие свойства. На основании значений модулей податливости для УАФ с кубической и гексагональной анизотропией была проанализирована изменчивость упругих „технических коэффициентов“ (модуля Юнга, коэффициента Пуассона и модуля сдвига). Модуль Юнга, коэффициент Пуассона и модуль сдвига для анизотропных материалов меняются с изменением ориентации оси растяжения относительно кристаллографических осей. В случае линейной упругости модуль Юнга $E(\mathbf{n})$ и коэффициент Пуассона $v(\mathbf{n}, \mathbf{m})$ зависят от тензорных модулей податливости $s_{i j k l}$, единичного вектора $\mathbf{n}$, направленного вдоль оси растяжения, и единичного вектора $\mathbf{m}$, перпендикулярного направлению растяжения [51]:

$$
\begin{gathered}
\frac{1}{E(\mathbf{n})}=s_{i j k l} n_{i} n_{j} n_{k} n_{l}, \\
v(\mathbf{n}, \mathbf{m})=-\frac{s_{i j k l} m_{i} m_{j} n_{k} n_{l}}{s_{\alpha \beta \lambda \mu} n_{\alpha} n_{\beta} n_{\lambda} n_{\mu}} .
\end{gathered}
$$

Модуль сдвига $G(\mathbf{n}, \mathbf{m})$ определяется вектором $\mathbf{n}$, являющимся единичным вектором нормали к плоскости скольжения, и единичным вектором m, указывающим направления скольжения [51]:

$$
G^{-1}(\mathbf{n}, \mathbf{m})=4 s_{i j k l} n_{i} m_{j} n_{k} m_{l} .
$$

Далее изменчивость „технических коэффициентов“ упругости вместо единичных векторов будем описывать тремя углами Эйлера: $\varphi, \theta, \psi$.

Упругость кубических кристаллов характеризуется тремя независимыми матричными модулями податливости: $s_{11}, s_{12}, s_{44}$. Тогда модуль Юнга, коэффициент Пуассона и модуль сдвига можно записать в виде $[37,52]$

$$
\begin{gathered}
\frac{1}{s_{11} E}=1-\frac{\delta}{2} M(\varphi, \theta), \\
\frac{v}{s_{11} E}=-\frac{\delta}{2}[N(\varphi, \theta, \psi)-\Pi], \\
\frac{1}{s_{44} G}=1+(A-1) N(\varphi, \theta, \psi), \\
\Pi \equiv-\frac{2 s_{12}}{\Delta}, \\
\delta \equiv \frac{\Delta}{s_{11}},
\end{gathered}
$$

где

$$
\begin{gathered}
A \equiv 2 \frac{s_{11}-s_{12}}{s_{44}}=2 \frac{c_{44}}{c_{11}-c_{12}}, \\
0 \leq M \equiv \sin ^{2} 2 \theta+\sin ^{4} \theta \sin ^{2} 2 \varphi \leq \frac{4}{3}, \\
0 \leq N \equiv 3 \sin ^{2} \theta \cos ^{2} \theta \cos ^{2} \psi \\
+(\cos \theta \cos 2 \varphi \cos \psi-\sin \psi)^{2} \sin ^{2} \theta \leq 1 .
\end{gathered}
$$

Комбинация коэффициентов податливости $\Delta \equiv s_{11}-s_{12}$ $-0.5 s_{44}$ известна как параметр анизотропии для кубических кристаллов, а $A-$ как коэффициент упругой анизотропии Зенера.
Таблица 2. Экстремальные значения модуля Юнга и параметра анизотропии $\Delta$

\begin{tabular}{c|r|r|r|r}
\hline УА $\Phi$ & $\begin{array}{c}\Delta, \\
\mathrm{TPa}^{-1}\end{array}$ & $\begin{array}{r}E_{[100]}, \\
\mathrm{GPa}\end{array}$ & $\begin{array}{c}E_{[110]}, \\
\mathrm{GPa}\end{array}$ & $\begin{array}{c}E_{[11],}, \\
\mathrm{GPa}\end{array}$ \\
\hline CA3 & 1.06 & 535 & 746 & $\mathbf{8 6 0}$ \\
CA4 & -2.51 & $\mathbf{5 3 5}$ & 320 & 282 \\
CA6 & -3.31 & $\mathbf{1 0 5 5}$ & 384 & 317 \\
CA7 & 9.85 & 123 & 313 & $\mathbf{6 4 4}$ \\
CA8 & -0.99 & $\mathbf{5 9 9}$ & 462 & 430 \\
CA9 & 0.98 & 268 & 308 & $\mathbf{3 2 5}$ \\
CB & 2.53 & 181 & 234 & $\mathbf{2 6 0}$
\end{tabular}

Анализ изменчивости модуля Юнга (10) позволяет определить три экстремальных значения [53], соответствующих растяжению в направлениях [100], [110] и [111]:

$$
\begin{gathered}
E_{[100]}=\frac{1}{s_{11}}, \\
E_{[110]}=\frac{1}{s_{11}-\Delta / 2}, \\
E_{[111]}=\frac{1}{s_{11}-2 \Delta / 3} .
\end{gathered}
$$

Экстремальные значения модуля Юнга зависят от знака и величины параметра анизотропии $\Delta$. Для кубических кристаллов с положительной анизотропией $\Delta>0$ из соотношений (15)-(17) имеем $E_{[111]}>E_{[110]}>E_{[100]}$. Для кубических кристаллов с отрицательной анизотропией $\Delta<0$ из этих соотношений следуют противоположные неравенства: $E_{[100]}>E_{[110]}>E_{[111]}$. На основе величин упругих констант равновесных структур с кубической анизотропией из углеродных алмазоподобных кластеров, представленных в табл. 1, определены экстремальные значения модуля Юнга и значения параметра анизотропии $\Delta$ (табл. 2). Три из равновесных алмазоподобных фаз с кубической структурой (СА4, СА6 и СА8), имеют отрицательную анизотропию, а четыре другие - положительную анизотропию. Наибольшие модули Юнга оказались у фазы СА6 (1055 GPa, $\Delta<0)$ при растяжении в направлении [100] и фазы СА3 (860 GPa, $\Delta>0)$ в направлении [111]. Жирным шрифтом в табл. 2 помечены максимальные значения модуля Юнга.

Основываясь на безразмерных параметрах П и $\delta$, (см. (13) и (14)), все кубические кристаллы можно поделить на три типа [37]: неауксетики (П > 1, $\delta>0$ и $\Pi<0, \delta<0)$, частичные ауксетики $(0<\Pi<1$ при $\delta>0$ и $\delta<0)$ и полные ауксетики $(\Pi<0, \delta>0$ и $\Pi>1, \delta<0)$. Неауксетики имеют положительный коэффициент Пуассона при любых ориентациях, а полные ауксетики - отрицательный коэффициент. У частичных ауксетиков возможны как положительные значения коэффициента Пуассона, так и отрицательные. Расчеты безразмерных параметров П и $\delta$ показывают, что две из восьми изученных равновесных алмазоподобных структур с кубической анизотропией СА3 $(\Pi=0.83)$ 
Таблица 3. Экстремальные значения коэффициентов Пуассона (глобальные максимумы и минимумы $v_{\max }, v_{\min }$, экстремумы при частных ориентациях $\left.v_{[100],[001]}, v_{[001],[110]}, v_{[110],[110]}, v_{(111),[111]}\right)$, а также усредненное по всем направлениям значение коэффициента Пуассона $\langle v\rangle$ и значения безразмерных параметров П и $\delta$

\begin{tabular}{c|c|c|c|c|c|c|c|c|c}
\hline УАФ & $\Pi$ & $\delta$ & $v_{\min }$ & $v_{\max }$ & $\langle v\rangle$ & $v_{[100],[001]}$ & $v_{[001],[110]}$ & $v_{[110],[110]}$ & $v_{(111),[111]}$ \\
\hline CA3 & 0.83 & 0.57 & -0.07 & 0.33 & 0.15 & 0.24 & 0.33 & -0.07 & 0.07 \\
CA4 & -0.348 & -1.34 & 0.14 & 0.54 & 0.32 & 0.23 & 0.14 & 0.54 & 0.36 \\
CA6 & -0.044 & -3.49 & 0.03 & 0.66 & 0.31 & 0.08 & 0.03 & 0.66 & 0.37 \\
CA7 & 0.739 & 1.21 & -0.40 & 1.14 & 0.38 & 0.45 & 1.14 & -0.40 & 0.23 \\
CA8 & -0.606 & -0.59 & 0.14 & 0.37 & 0.24 & 0.18 & 0.14 & 0.37 & 0.27 \\
CA9 & 1.840 & 0.26 & 0.13 & 0.28 & 0.21 & 0.24 & 0.28 & 0.13 & 0.19 \\
CВ & 1.584 & 0.46 & 0.17 & 0.47 & 0.33 & 0.36 & 0.47 & 0.17 & 0.30
\end{tabular}

и СА7 (П = 0.739) могут иметь отрицательный коэффициент Пуассона (табл. 3), т. е. эти алмазоподобные фазы являются частичными ауксетиками. Полных ауксетиков среди исследуемых алмазоподобных фаз не обнаружено. В табл. 3 помимо значений безразмерных параметров П и $\delta$ приведены глобальные максимальные и минимальные значения коэффициентов Пуассона $\left(v_{\max }, v_{\min }\right)$, усредненное по всем направлениям значение коэффициента Пуассона $\langle v\rangle$. Наименьший коэффициент Пуассона выявляется у равновесной структуры СА7 $\left(v_{\min }=-0.4\right)$. Для этого ауксетика имеется большой диапазон изменения коэффициента Пуассона $\left(v_{\max }-v_{\min }=1.54\right)$. Средний коэффициент Пуассона $\langle v\rangle$ для всех представленных кристаллических материалов оказывается положительным и меняется в пределах от 0.15 до 0.38 . Оценим также значения коэффициентов Пуассона для некоторых частных ориентаций, которые определяются по формулам

$$
\begin{gathered}
v_{[100],[001]}=-\frac{s_{12}}{s_{11}}, \\
v_{[001],[110]}=-\frac{s_{12}}{s_{11}-0.5 \Delta}, \\
v_{[1 \overline{1} 0],[110]}=-\frac{2 s_{12}+\Delta}{2 s_{11}-\Delta}, \\
v_{(111),[111]}=-\frac{3 s_{12}+\Delta}{3 s_{11}-2 \Delta} .
\end{gathered}
$$

Последние три числа в квадратных скобках указывают направления растяжения, а первые три числа в скобках - направление поперечной деформации. Коэффициенты Пуассона можно выразить через безразмерные параметры П и $\delta$

$$
\begin{gathered}
v_{[100],[001]}=\frac{\Pi \delta}{2}, \\
v_{[001],[110]}=\frac{\Pi \delta}{2-\delta}, \\
v_{[1 \overline{1} 0],[110]}=\frac{\delta(\Pi-1)}{2-\delta}, \\
v_{(111)[111]}=\frac{\delta(1.5 \Pi-1)}{3-2 \delta} .
\end{gathered}
$$

Численно-аналитический анализ этих формул с использованием термодинамических ограничений П $\delta>2 \delta-2$, $1>\Pi \delta>-2, \delta<1.5$ [37] показывает, что при П $>0,0<\delta<1.5$

$$
v_{[001],[110]}>v_{[100],[001]}>v_{(111)[111]}>v_{[1 \overline{1} 0],[110]},
$$

при $\Pi>0, \delta<0$

$$
v_{[1 \overline{1} 0],[110]}>v_{(111)[111]}>v_{[001],[110]}>v_{[100],[001]},
$$

при $\Pi<0, \delta<0$

$$
v_{[1 \overline{1} 0],[110]}>v_{(111)[111]}>v_{[100],[001]}>v_{[001],[110]},
$$

при $\Pi<0,0<\delta<1.5$

$$
v_{[100],[001]}>v_{[001],[110]}>v_{(111)[111]}>v_{[11 \overline{1} 0],[110]} .
$$

Как видно из табл. 3, значения коэффициентов Пуассона при частных ориентациях для равновесных алмазоподобных структур удовлетворяют указанным неравенствам. Для структур СА3 и СА7 отрицательные значения будут наблюдаться у коэффициента $v_{[1 \overline{1} 0],[110]}$.

На рис. 2 показаны поверхности ауксетичности $v(\varphi, \theta, \psi)=0$ для алмазоподобных фаз СА3 и СА7, которые были построены в пространстве углов Эйлера с периодами $T_{\varphi}=\pi / 2, T_{\theta}=2 \pi$ и $T_{\psi}=\pi$. Для этих фаз ауксетичность имеет место внутри представленных поверхностей. По этим поверхностям можно определить углы Эйлера, т.е. направления растяжения, при которых будет наблюдаться отрицательный коэффициент Пуассона. Как видно из рис. 2, наибольшей зоной ауксетичности будет обладать кристаллический материал СА7.

Анализ формулы модуля сдвига (12) позволяет определить максимальное и минимальное значения [52]

$$
\begin{gathered}
G_{1}=\frac{1}{s_{44}}, \\
G_{2}=\frac{1}{2\left(s_{11}-s_{12}\right)} .
\end{gathered}
$$

Какое из экстремальных значений модуля сдвига окажется максимальным, а какое минимальным, зависит от величины коэффициента анизотропии Зенера $A$

$$
A \equiv 2 \frac{s_{11}-s_{12}}{s_{44}}=1+\frac{2 \Delta}{s_{44}} .
$$


Таблица 4. Максимальные и минимальные значения модуля сдвига, коэффициента анизотропии Зенера $A$ и объемного модуля $B$

\begin{tabular}{|c|c|c|c|c|c|c|}
\hline \multirow{2}{*}{ УАФ } & \multirow{2}{*}{$A$} & \multirow{2}{*}{$G_{1}=s_{44}^{-1}, \mathrm{GPa}$} & \multirow{2}{*}{$G_{2}=\left(2\left(s_{11}-s_{12}\right)\right)^{-1}, \mathrm{GPa}$} & \multicolumn{3}{|c|}{$B, \mathrm{GPa}$} \\
\hline & & & & Наст. раб. & {$[1,8]$} & {$[13]$} \\
\hline CA3 & 1.85 & 400 & 216 & 337 & 342 & 346.2 \\
\hline CA4 & 0.48 & 104 & 217 & 335 & 249 & 284.5 \\
\hline CA6 & 0.24 & 115 & 490 & 415 & 323 & 350.5 \\
\hline $\mathrm{CA} 7$ & 6.16 & 262 & 43 & 398 & 207 & 269.6 \\
\hline CA8 & 0.67 & 169 & 254 & 311 & - & 274.5 \\
\hline CA9 & 1.27 & 137 & 108 & 173 & - & 239.2 \\
\hline $\mathrm{CB}$ & 1.50 & 100 & 66 & 218 & - & 277.6 \\
\hline
\end{tabular}

Для кубических кристаллов с положительной анизотропией $\Delta>0$ (или $A>1$ ) из соотношений (26) и (27) имеем $G_{1}>G_{2}$. Для кубических кристаллов с отрицательной анизотропией $\Delta<0$ (или $0<A<1$ ) следует
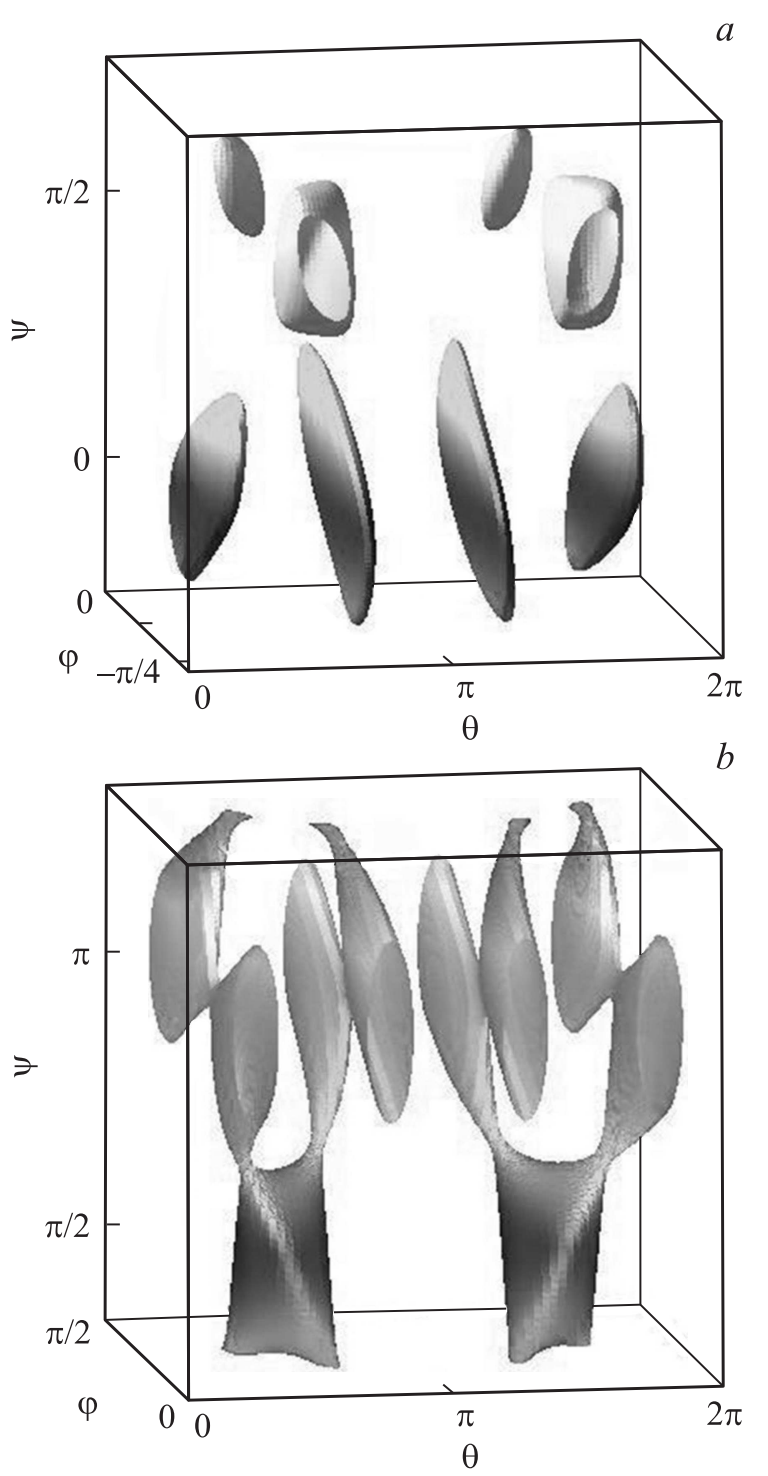

Pис. 2. Поверхности ауксетичности углеродных алмазоподобных структур СА3 $(a)$ и CA7 $(b)$ с кубической анизотропией. противоположное неравенство: $G_{1}<G_{2}$. В табл. 4 представлены значения коэффициента анизотропии Зенера $A$, экстремальные значения для модуля сдвига и значения объемного модуля, определяемого по формуле $B=\left(c_{11}+2 c_{12}\right) / 3$. Наибольший модуль сдвига оказывается у алмазоподобной фазы СА6 (490 GPa) при экстремальном значении $G_{2}$. Наименьший модуль сдвига наблюдается у структуры CA7 $\left(G_{2}=43 \mathrm{GPa}\right)$. Наибольший объемный модуль выявляется у равновесной структуры СА6 (415 GPa), а наименьший - у структуры CA9 (173 GPa).

Для гексагональных кристаллов модуль Юнга (7), коэффициент Пуассона (8) и модуль сдвига (9) можно записать в виде [54]

$$
\begin{gathered}
\frac{1}{E}=s_{11}+\left(s_{33}-s_{11}-\delta_{0} \sin ^{2} \theta\right) \cos ^{2} \theta \\
-\frac{v}{E}=s_{13}+\left(\left(s_{12}-s_{13}\right) \sin ^{2} \psi+\delta_{0} \cos ^{2} \theta \cos ^{2} \psi\right) \sin ^{2} \theta \\
\frac{1}{G}=s_{44}+\left(\left(2 s_{11}-2 s_{12}-s_{44}\right) \sin ^{2} \psi\right. \\
\left.+4 \delta_{0} \cos ^{2} \theta \cos ^{2} \psi\right) \sin ^{2} \theta \\
\delta_{0} \equiv s_{11}+s_{33}-2 s_{13}-s_{44}
\end{gathered}
$$

Зависимости обсуждаемых модулей и коэффициента Пуассона являются периодическими функциями угловых переменных с периодами $T_{\theta}=T_{\psi}=\pi$. Упругие „технические коэффициенты“ для гексагональных кристаллов в отличие от случая кубических кристаллов зависят от меньшего количества углов. Модуль Юнга зависит только от одного угла Эйлера $(\theta)$, а модуль сдвига и коэффициент Пуассона - от двух углов $(\theta$ и $\psi)$.

На рис. 3, $а$ представлена поверхность коэффициента Пуассона для алмазоподобной фазы СА2, которая оказывается частичным ауксетиком. Минимальное значение коэффициента Пуассона получается при растяжении в направлении [010] и равно $v_{\min }=v_{[00 \overline{1}],[010]}=-s_{12} / s_{11}$ $=-0.04$. Другое экстремальное значение коэффициента при растяжении в направлении [010] принимает значение $v_{[100],[110]}=-s_{13} / s_{11}=0.14$. Максимальное значение коэффициента Пуассона $\left(v_{\max }=0.67\right)$ достигается при $\theta=0$ и $\psi=42,138^{\circ}$. При растяжении в 

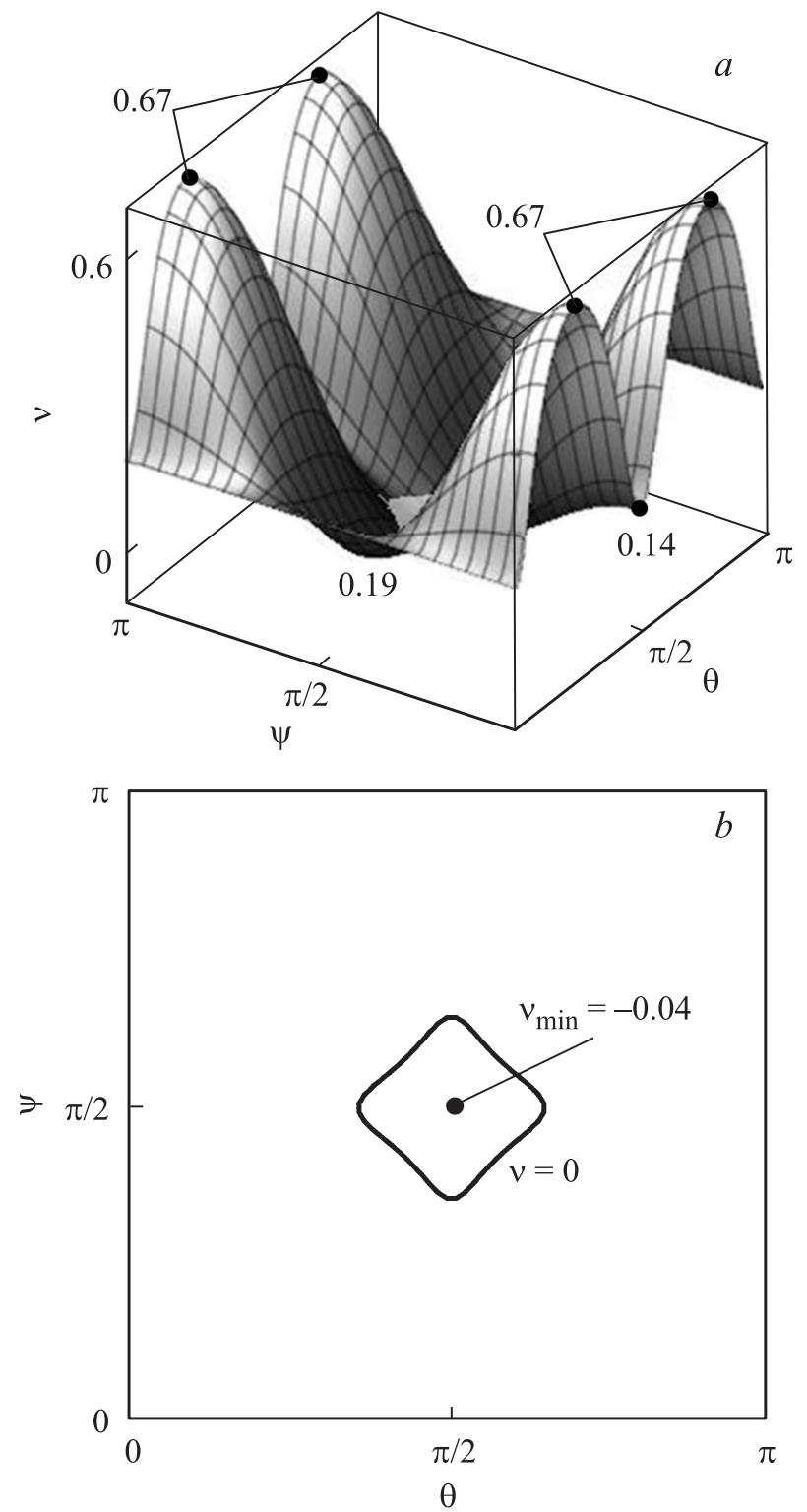

Рис. 3. Поверхность коэффициента Пуассона $(a)$ и кривая ауксетичности в пространстве двух углов Эйлера $(b)$ для равновесной алмазоподобной структуры СА2 с гексагональной анизотропией.

направлении [001] экстремальное значение коэффициента Пуассона равно $v_{(001),[001]}=-s_{13} / s_{33}=0.19$. Усредненное по всем направлениям значение коэффициента Пуассона $\langle v\rangle$ принимает значение 0.25 . На рис. $3, b$ представлена кривая ауксетичности, построенная по формуле

$$
s_{13}+\left(\left(s_{12}-s_{13}\right) \sin ^{2} \psi+\delta_{0} \cos ^{2} \theta \cos ^{2} \psi\right) \sin ^{2} \theta=0 .
$$

Зона ауксетичности находится внутри области, представленной на рисунке.

Изменчивость модуля Юнга для алмазоподобной структуры СА2 иллюстрируется рис. 4, на котором отражены также значения экстремальных значений модуля Юнга. Максимальное значение $521 \mathrm{GPa}$ достигается при растяжении в направлении [001]. Минимальное значение модуля Юнга $E_{\min }=181 \mathrm{GPa}$ наблюдается при $\psi=46$ и $134^{\circ}$.

Поверхность модуля сдвига для алмазоподобной структуры СА2 изображена на рис. 5. Значения модуля сдвига меняются от $54.4 \mathrm{GPa} \mathrm{при} \theta=0$ и любом $\psi$, что соответствует плоскости скольжения (100), до $206 \mathrm{GPa}$ при $\theta=\pi / 2$ и $\psi=\pi / 2$, что соответствует плоскости скольжения (010) в направлении [001]. Объемный модуль $B=\left(2 c_{11}+c_{33}+2 c_{12}+4 c_{13}\right) / 9$ для алмазоподобной фазы СА2 равен $87.1 \mathrm{GPa}$. Это значение оказывается одним из самых маленьких при сравнении со значениями объемного модуля для алмазоподобных фаз с кубической анизотропией (табл. 4).

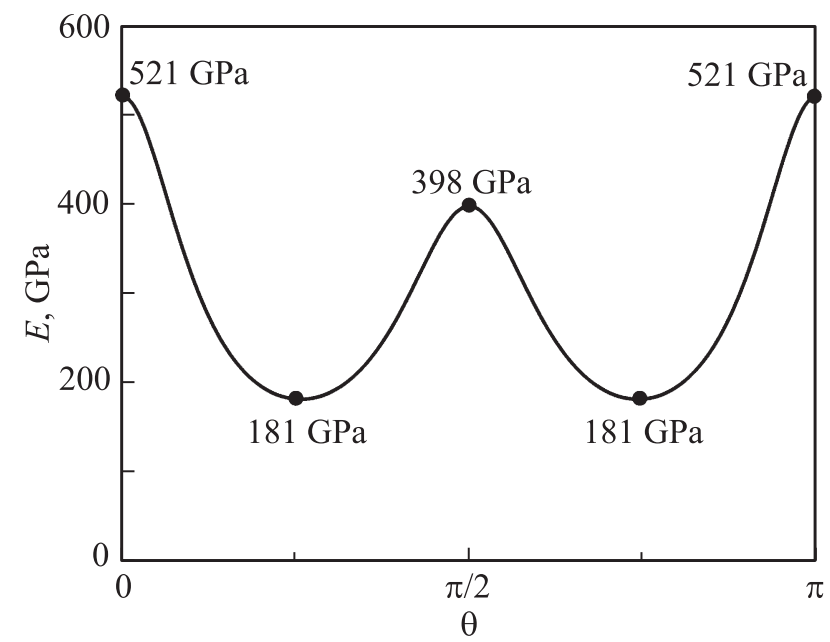

Рис. 4. Изменчивость модуля Юнга равновесной алмазоподобной структуры CA2 с гексагональной анизотропией. Точками отмечены экстремальные значения модуля Юнга.

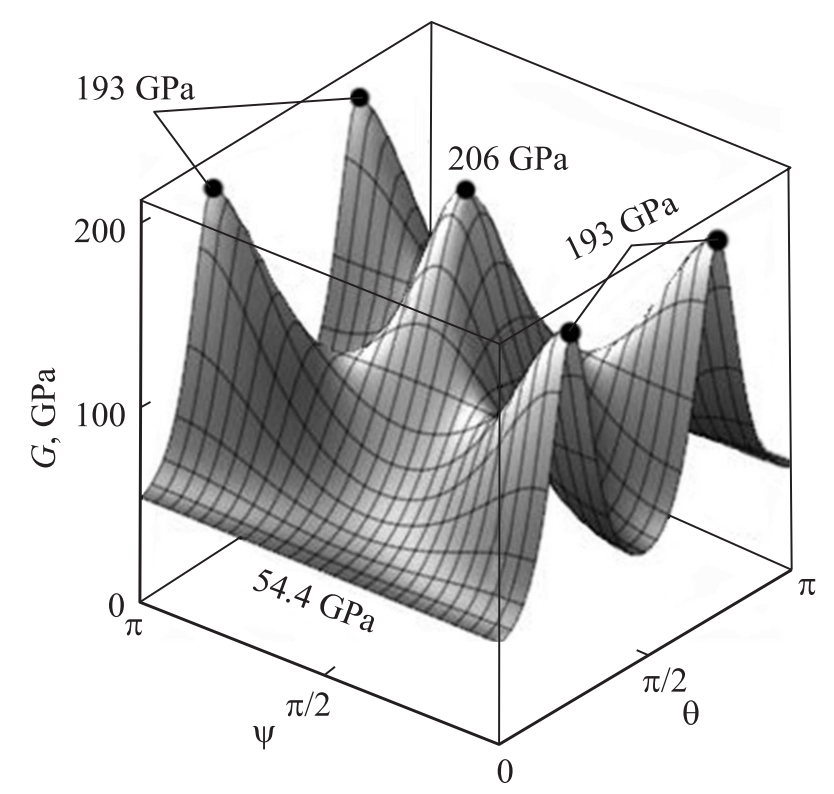

Рис. 5. Поверхность модуля сдвига для равновесной алмазоподобной структуры СА2 с гексагональной анизотропией. 
Таблица 5. Экспериментальные данные (при нормальных условиях) для кубического алмаза

\begin{tabular}{l|c|c|c|c}
\hline$c_{11}, \mathrm{GPa}$ & $c_{12}, \mathrm{GPa}$ & $c_{44}, \mathrm{GPa}$ & $B, \mathrm{GPa}$ & Лит. ссылка \\
\hline 1079 & 124 & 578 & 442 & {$[59]$} \\
1076.4 & 125.2 & 577.4 & 442.3 & {$[60]$} \\
1080.4 & 127 & 576.6 & 444.8 & {$[61]$} \\
1080.4 & 127 & 576.6 & 443.9 & {$[62]$}
\end{tabular}

\section{4. Заключение}

Равновесные алмазоподобные фазы СА1, СА2, СА3, CA4, CA5, СА6, СА7, СА8, СА9 и СВ, полученные сшивкой фуллереноподобных молекул, были исследованы методом молекулярной динамики. Среди названных алмазоподобных фаз восемь (CA1, СА3, CA4, CA6, CA7, CA8, СА9 и СВ) являются кубическими. Структура СА2 имеет гексагональную анизотропию, а структура СА5 - тетрагональную. Равновесная фаза СА5 оказалась неустойчивой, фаза СА1 также малоустойчива, в то время как все прочие фазы устойчивы по отношению к малой упругой деформации. Результаты расчета напряжений и упругих констант позволили проанализировать упругие характеристики (модуль Юнга, коэффициент Пуассона, модуль сдвига и объемный модуль) алмазоподобных фаз на основе кластеров фуллереноподобных молекул. Обнаружено, что три из десяти рассмотренных алмазоподобных фаз, а именно СA2, CA3 и СА7, являются ауксетиками. Поскольку коэффициент Пуассона у них оказывается отрицательным только в определенных направлениях, данные структуры являются частичными ауксетиками.

УАФ представляют собой новые перспективные материалы из $s p^{3}$-гибридизированных атомов, и настоящая работа вносит вклад в изучение их механических свойств. При этом поиск материалов-ауксетиков является важной задачей [23,55-58], поскольку они могут использоваться для разработки композитных материалов с заданными упругими свойствами. Нередко аномалии упругих свойств материалов бывают связаны с аномалиями их коэффициента теплового расширения, расчет которого предполагается провести в следующих работах.

В табл. 4 для сравнения приведены также численные значения объемного модуля для таких же алмазоподобных фаз, рассчитанные полуэмпирическими квантовомеханическими методами в $[1,8,13]$. Видны существенные количественные различия последних с полученными в настоящей работе методом молекулярной динамики значениями $B$, что, по-видимому, связано с большим различием использованных расчетных методов.

Для оценки полученных выше механических характеристик алмазоподобных фаз, собранных в табл. 1 и 4, в табл. 5 приводятся экспериментальные данные для кубического алмаза из работ [59-62]. Из сравнения можно видеть, что объемный модуль кубического алмаза $(\sim 440 \mathrm{GPa})$ превосходит объемные модули всех рассмотренных алмазоподобных фаз. Наименьшее различие величин объемного модуля имеется с фазами СА3,
CA6 и CA7. Что касается коэффициента жесткости $c_{11}$, то наблюдается удивительная близость его значений для алмазоподобной фазы СА6 и кубического алмаза (табл. 1 и 5 ).

\section{Список литературы}

[1] В.А. Грешняков, Е.А. Беленков, В.М. Березин. Кристаллическая структура и свойства углеродных алмазоподобных фаз. Изд. центр ЮУрГУ, Челябинск (2012). 150 с.

[2] В.А. Плотников, Д.Г. Богданов, С.В. Макаров. Детонационный наноалмаз. Изд-во Алтай. гос. ун-та, Барнаул (2014). $224 \mathrm{c}$.

[3] В.В. Покропивный, А.В. Покропивный. ФТТ 46, 2, 380 (2004).

[4] В.Л. Бекенев, В.В. Покропивный. ФТТ 48, 7, 1324 (2006).

[5] J. Crain, S.J. Clark, G.J. Ackland, M.C. Payne, V. Milman, P.D. Hatton, B.J. Reid. Phys. Rev. B 49, 8, 5329 (1994).

[6] Е.А. Беленков, В.А. Грешняков. ФТТ 55, 8, 1640 (2013).

[7] Е.А. Беленков, В.А. Грешняков. ФТТ 57, 1, 192 (2015).

[8] В.А. Грешняков, Е.А. Беленков. ЖЭТФ 140, 1, 99 (2011)

[9] Е.А. Беленков, В.А. Грешняков. ФТТ 57, 6, 229 (2015).

[10] H.S. Domingos. J. Phys.: Condens. Matter. 16, 9083 (2004).

[11] Е.А. Беленков, И.В. Шахова. ФТТ 53, 11, 2265 (2011).

[12] Е.А. Беленков, А.Е. Коченгин. ФТТ 57, 10, 2071 (2015).

[13] Е.А. Беленков, В.А. Грешняков. ФТТ 57, 11, 2262 (2015).

[14] E.A. Belenkov, M.M. Brzhezinskaya, V.A. Greshnyakov. Diamond. Rel. Mater. 50, 9 (2014).

[15] Е.А. Беленков, В.А. Грешняков. ЖЭТФ 146, 1, 116 (2014).

[16] Р.Р. Мулюков, Ю.А. Баимова. Углеродные наноматериалы. РИЦ БашГУ, Уфа. (2015). 160 с.

[17] К.А. Крылова, Ю.А. Баимова, С.В. Дмитриев, Р.Р. Мулюков. ФТТ 58, 2, 384 (2016).

[18] R.K. Roy, K.-R. Lee. J. Biomed. Mater. Res. B 83, 1, 72 (2007).

[19] X. Li, D.H.C. Chua. In: Ceramic integration and joining technologies: from Macro to nanoscale / Eds M. Singh, T. Ohji, R. Asthana, S. Mathur. John Wiley and Sons, Inc., Hoboken, NJ, USA (2011). P. 641.

[20] W.I. Milne. Semicond. Sci. Technol. 18, S81 (2003).

[21] J. Robertson. Mater. Sci. Eng. R 37, 129 (2002).

[22] G. Van Lier, C. Van Alsenoy, V. Van Doren, P. Geerligs. Chem. Phys. Lett. 326, 181 (2000).

[23] F. Scarpa, S. Adhikari, C.Y. Wang. J. Phys. D 42, 142002 (2009).

[24] A. Smolyanitsky, V.K. Tewary. Nanotechnology 22, 085703 (2011).

[25] R.H. Baughman, J.M. Shacklette, A.A. Zakhidov, S. Stafstrom. Nature 392, 362 (1998).

[26] S.P. Tokmakova. Phys. Status Solidi B 242, 721 (2005).

[27] A. Norris. Proc. Roy. Soc. A 462, 3385 (2006).

[28] T. Paszkiewicz, S. Wolski. Phys. Status Solidi B 244, 966 (2007).

[29] T. Paszkiewicz, S. Wolski. J. Phys.: Conf. Ser. 104, 012038 (2008).

[30] A.C. Branka, D.M. Heyes, K.W. Wojciechowski. Phys. Status Solidi B 246, 2063 (2009).

[31] Р.В. Гольдштейн, В.А. Городцов, Д.С. Лисовенко. Изв. PAH. MTT 4, 43 (2010).

[32] Р.В. Гольдштейн, В.А. Городцов, Д.С. Лисовенко. ДАН. 439, 2, 184 (2011). 
[33] A.C. Branka, D.M. Heyes, K.W. Wojciechowski. Phys. Status Solidi B 248, 96 (2011).

[34] A.C. Branka, D.M. Heyes, Sz. Mackowiak, S. Pieprzyk, K.W. Wojciechowski. Phys. Status Solidi B 249, 1373 (2012).

[35] R.V. Goldstein, V.A. Gorodtsov, D.S. Lisovenko. Phys. Status Solidi B 250, 10, 2038 (2013).

[36] V.V. Krasavin, A.V. Krasavin. Phys. Status Solidi B 251, 2314 (2014).

[37] Р.В. Гольдштейн, В.А. Городцов, Д.С. Лисовенко, М.А. Волков. Физ. мезомеханика 16, 6, 13 (2013).

[38] Р.В. Гольдштейн, В.А. Городцов, Д.С. Лисовенко, М.А. Волков. Письма о материалах 5, 4, 409 (2015).

[39] Н.П. Кобелев, Р.К. Николаев, Я.М. Сойфер, С.С. Хасанов. ФТТ 40, 1, 173 (1998).

[40] Н.П. Кобелев. ФТТ 44, 1, 173 (2002).

[41] Н.П. Кобелев, Р.К. Николаев, Н.С. Сидоров, Я.М. Сойфер. ФТT 44, 3, 415 (2002).

[42] Н.П. Кобелев, Р.К. Николаев, Н.С. Сидоров, Я.М. Сойфер. ФTT 43, 12, 2244 (2001).

[43] http://lammps.sandia.gov/

[44] S. Stuart, A. Tutein, J. Harrison. J. Chem. Phys. 112, 6472 (2000).

[45] W. Brenner. Phys. Rev. B 42, 9458 (1992).

[46] A. K. Singh, R.G. Hennig. Phys. Rev. B 87, 094112 (2013).

[47] S. Costamagna, M. Neek-Amal, J.H. Los, F.M. Peeters. Phys. Rev. B 86, 041408 (2012).

[48] J.A. Baimova, B. Liu, S.V. Dmitriev, N. Srikanth, K. Zhou. Phys. Chem. Chem. Phys. 16, 19505 (2014).

[49] Ю.А. Баимова, Р.Т. Мурзаев, С.В. Дмитриев. ФТТ 56, 10 , 1946 (2014).

[50] Л.Х. Рысаева, Ю.А. Баимова. Фундаментальные проблемы современного материаловедения 12, 4, 439 (2015).

[51] Ю.И. Сиротин, М.П. Шаскольская. Основы кристаллофизики. Наука, М. (1975). 680 с.

[52] Р.В. Гольдштейн, В.А. Городцов, Д.С. Лисовенко. Письма о материалах 2, 1, 21 (2012).

[53] Р.В. Гольдштейн, В.А. Городцов, Д.С. Лисовенко. Письма о материалах 1, 3, 127 (2011).

[54] Р.В. Гольдштейн, В.А. Городцов, Д.С. Лисовенко. ДАН 441, 4, 468 (2011).

[55] K.W. Wojciechowski. Phys. Lett. A 137, 1-2, 60 (1989).

[56] K.V. Tretiakov, K.W. Wojciechowski. Phys. Status Solidi B 250, 2020 (2013).

[57] J.N. Grima, S. Winczewski, L. Mizzi, M.C. Grech, R. Cauchi, R. Gatt, D. Attard, K.W. Wojciechowski. J. Rybicki. Adv. Mater. 27, 8, 1455 (2015).

[58] K.L. Alderson, A. Alderson, J.N. Grima, K.W. Wojciechowski. Phys. Status Solidi B 251, 263 (2014).

[59] H.J. McSkimin, P. Andreatch. J. Appl. Phys. 43, 2944 (1972).

[60] M. Grimsditch, A.K. Ramdas. Phys. Rev. B 11, 3139 (1975).

[61] E.S. Zouboulis, M. Grimsditch, A.K. Ramdas, S. Rodrigues. Phys. Rev. B 57, 2889 (1998).

[62] A. Migliori, H. Ledbetter, R.G. Leisure, J.B. Betts. J. Appl. Phys. 104, 053512 (2008). 